\title{
SEMIORTHOGONAL LINEAR PREWAVELETS ON IRREGULAR MESHES
}

\author{
PETER OSWALD \\ School of Engineering and Science, International University Bremen \\ Campus Ring 1, D-28759 Bremen, Germany \\ E-mail:p.oswald@iu-bremen.de
}

Dedicated to Professor Zbigniew Ciesielski

\begin{abstract}
We extend results on constructing semiorthogonal linear spline prewavelet systems in one and two dimensions to the case of irregular dyadic refinement. In the one-dimensional case, we obtain sharp two-sided inequalities for the $L_{p}$-condition, $1<p<\infty$, of such systems.
\end{abstract}

1. Introduction. This note is devoted to the $L_{p}$-stability of semiorthogonal linear spline prewavelet systems in one and two dimensions. On regular simplicial partitions resp. for semiregular refinement of arbitrary partitions, such prewavelet systems have been studied to great extent, see $[2,8,3,14,9]$ resp. [15, 4, 5]. Our interest in the case of irregular dyadic refinement is triggered by recent attempts to theoretically investigate nonlinear approximation processes and multiresolution analyses where the underlying spatial grid structures are less regular. Although the restriction to the linear spline case allows for some simplifications, its separate treatment provides useful insights, and is also justified by a number of concrete applications to irregular sampling, surface discretization, image analysis, empirical density function estimation, and to the numerical solution of partial differential equations by adaptive finite element methods.

Similar studies have recently been undertaken for orthogonal spline systems (in particular, for the Franklin system) the systematic investigation of which was pioneered in the early 1970ies by Zbigniew Ciesielski. E.g., Ciesielski, Gevorkjan, and Kamont [1, 6, 7] have considered Franklin systems on arbitrary 1D partition sequences, and obtained a number of general results. In particular, in [7] $L_{p}$-unconditionality $(1<p<\infty)$ of Franklin systems is shown to hold, independently of the grid refinement process. For generalizations to higher dimensions, see Petrushev [13] and the papers cited therein. Lyche, Morken,

2000 Mathematics Subject Classification: 41A15, 42C60, 65N30.

The paper is in final form and no version of it will be published elsewhere. 
and Quak [10] have independently introduced prewavelet constructions on arbitrary 1D partition sequences.

In this paper, semiorthogonal prewavelet systems $\boldsymbol{\Psi}$ are constructed following the standard lifting scheme used by Stevenson et al. [15, 4] for the semiregular refinement case. For the $1 \mathrm{D}$ case, we present necessary and sufficient conditions for $L_{p}$-stability of $\boldsymbol{\Psi}$ in Proposition 5 (some weaker results have independently been obtained by Mikkelsen, Oja, and Quak $[11,12])$. In 2D, where the results are still incomplete, two quantities seem to matter: the maximal valence of vertices in the coarse partition (this quantity depends only on the initially given triangulation since all new vertices have valence 6 ), and an upper bound for the ratios $\left|\operatorname{supp} \tilde{\phi}_{Q}\right| /\left|\operatorname{supp} \tilde{\phi}_{P}\right|$ of the support areas of fine grid nodal basis functions associated with new vertices $Q$ in a small neighborhood of an old vertex $P$. The above quantities enter the estimates for the stability constants to the power $p-1$, thus, for $p=1$ we have unconditional stability.

2. Notation and definitions. We will consistently use the following notation:

- Throughout this paper, $L_{p}$-spaces are defined on $\mathbb{R}^{d}$, where $d \leq 2$. The $L_{p}$-norm of a function $f \in L_{p}$ is denoted by $\|f\|_{p}$. We also need scalar products

$$
(f, g)=\int_{\mathbb{R}^{d}} f(x) g(x) d x
$$

which are well-defined in the appearances below.

- A set $F=\left\{f_{i}\right\} \subset L_{p}$ is called $L_{p}$-stable if there exist constants $0<C_{1}<C_{2}<\infty$ such that for all sequences $\left\{x_{i}\right\}$ the following two-sided inequality holds:

$$
C_{1} \sum_{i}\left|x_{i}\right|^{p}\left\|f_{i}\right\|_{p}^{p} \leq\left\|\sum_{i} x_{i} f_{i}\right\|_{p}^{p} \leq C_{2} \sum_{i}\left|x_{i}\right|^{p}\left\|f_{i}\right\|_{p}^{p} .
$$

This assumes that the summation $\sum_{i} x_{i} f_{i}$ makes sense which is always the case for the systems under consideration. The optimal constants $C_{1}, C_{2}$ in (1) will be called lower and upper $L_{p}$-stability constants of $F$. Note that the weights $\left\|f_{i}\right\|_{p}^{p}$ are a natural choice since if (1) holds with $\left\|f_{i}\right\|_{p}^{p}$ replaced by arbitrary weights $\mu_{i}$ then taking the coordinate sequences as $\left\{x_{i}\right\}$ shows that $C_{1} \mu_{i} \leq\left\|f_{i}\right\|_{p}^{p} \leq C_{2} \mu_{i}$ for all $i$. This shows that if $L_{p}$-stability holds with any weighted $\ell_{p}$ coefficient norm then it also holds with the weights given in (1). Consequently, unless we are interested in the best possible stability constants resp. in the value of the $L_{p}$-condition of $F$ $\kappa_{p}(F)=\inf C_{2} / C_{1}$, there is no point in talking about a better choice of weights. For the remainder of this paper, we will stick to the definition (1). The counterpart of (1) for $p=\infty$ reads

$$
C_{1} \sup _{i}\left|x_{i}\right|\left\|f_{i}\right\|_{\infty} \leq\left\|\sum_{i} x_{i} f_{i}\right\|_{\infty} \leq C_{2} \sup _{i}\left|x_{i}\right|\left\|f_{i}\right\|_{\infty},
$$

but, although our results below can easily be extended to $p=\infty$, we will not go into this case.

- $\mathcal{T}$ and $\tilde{\mathcal{T}}$ stand for coarse and dyadically refined fine simplicial partitions of $\mathbb{R}^{d}$ (the case of bounded polyhedral domains requires some boundary treatment which can be handled by an extension procedure, we will avoid these technicalities), $\mathcal{V}$ and 
$\tilde{\mathcal{V}}$ are the corresponding sets of vertices. We will consistently use the letter $P$ to indicate that $P$ is an old vertex (i.e., $P \in \mathcal{V}$ ), the letter $Q$ is used for new vertices $(Q \in \tilde{\mathcal{V}} \backslash \mathcal{V})$, and $R$ is used to denote a generic vertex (old or new) in $\tilde{\mathcal{V}}$. Note that dyadic refinement means that each edge in $\mathcal{T}$ carries exactly one $Q$, and that for $d=2$ the new $\tilde{\mathcal{T}}$ is obtained by inserting new edges connecting the $Q$ belonging to the same triangle in $\mathcal{T}$. If the position of $Q$ is restricted to the edge midpoint, this dyadic refinement is called semiregular, otherwise, if there are no restrictions on the placement of $Q$ on the edges, we call the dyadic refinement irregular. Throughout this paper, we always have in mind irregular dyadic refinement.

- $V$ and $\tilde{V}$ are the linear spline spaces on these partitions, their standard bases (consisting of hat functions of unit $L_{\infty}$ norm associated with the vertices) are denoted by $\boldsymbol{\Phi}=\left\{\phi_{P}\right\}$ and $\tilde{\boldsymbol{\Phi}}:=\left\{\tilde{\phi}_{R}\right\}$. By $\tilde{\Delta}_{R}$ we denote the area of the support of $\tilde{\phi}_{R}$ (the 1-ring of simplices around $R$ in the fine partition). Recall that nodal bases are unconditionally $L_{p}$-stable, i.e.,

$$
\left\|\sum_{R} c_{R} \tilde{\phi}_{R}\right\|_{p}^{p} \asymp \sum_{R}\left|c_{R}\right|^{p}\left\|\tilde{\phi}_{R}\right\|_{p}^{p} \asymp \sum_{R}\left|c_{R}\right|^{p} \tilde{\Delta}_{R}, \quad 1 \leq p<\infty,
$$

where $\asymp$ stands for a two-sided inequality, with constants that depend on $p$, at most, but are independent of $\mathcal{T}, \tilde{\mathcal{T}}$ (in the following, the letters $c, C$ are used for generic (positive) constants which may change from formula to formula, and generally depend on $p$ but not on any other quantities involved).

- In our considerations, the 1-ring in $\mathcal{T}$ of an arbitrary $P \in \mathcal{V}$ is of special interest. Figure 1 a) shows the notation for $d=2$. The number $k \equiv k_{P}$ of simplices attached
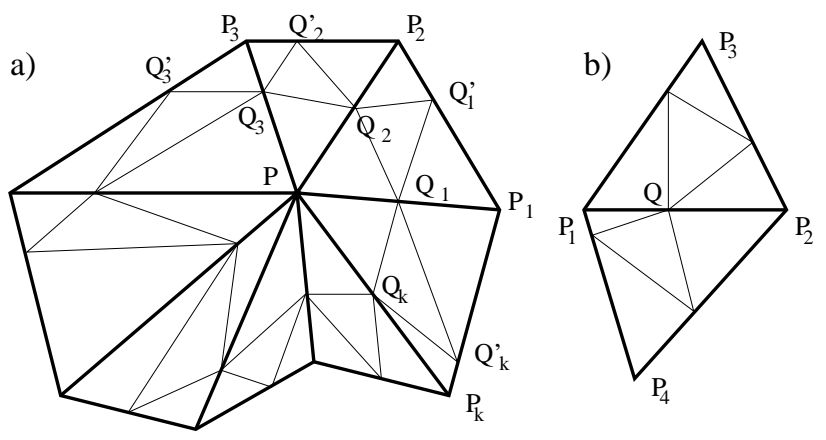

Fig. 1. Notation for 1-ring around $P$, and $P$-neighborhood of $Q$

to $P$ is called valence of $P$. We define the $Q$-neighborhood $\tilde{\mathcal{V}}_{P}$ of $P \in \mathcal{V}$ and its subset $\tilde{\mathcal{V}}_{P}^{*}$ as

$$
\tilde{\mathcal{V}}_{P}=\tilde{\mathcal{V}}_{P}^{*} \cup\left\{Q_{l}^{\prime}: l=1, \ldots, k\right\}, \quad \tilde{\mathcal{V}}_{P}^{*}=\left\{Q_{l}: l=1, \ldots, k\right\} .
$$

Finally, we define a $P$-neighborhood of $Q \in \tilde{\mathcal{V}} \mathcal{V}$ by setting

$$
\mathcal{V}_{Q}=\left\{P \in \mathcal{V}: Q \in \tilde{\mathcal{V}}_{P}\right\} .
$$

For $d=2$, this neighborhood always consists of 4 vertices (see Figure $1 \mathrm{~b}$ )) while $\# \tilde{\mathcal{V}}_{P}=2 k$, and $\# \tilde{\mathcal{V}}_{P}^{*}=k$ depend on the valence. For $d=1$, we have always $k_{P}=2$, 
$\tilde{\mathcal{V}}_{P}=\tilde{\mathcal{V}}_{P}^{*}$ consists of the two new vertices $Q^{ \pm}$inserted into the two intervals attached to the left and right of $P$, and $\mathcal{V}_{Q}$ consists of two old vertices $P^{ \pm}$(the left and right endpoints of the interval in $\mathcal{T}$ containing $Q$ ).

3. Properties of the dual system. We call $\boldsymbol{\Theta}=\left\{\theta_{P}: P \in \mathcal{V}\right\} \subset \tilde{V}$ a dual system for the nodal basis $\boldsymbol{\Phi}$ if for all $P \in \mathcal{V}$

$$
\left(\theta_{P}, \phi_{P^{\prime}}\right)=0 \quad \forall P^{\prime} \neq P, \quad\left(\theta_{P}, \phi_{P}\right)=1 .
$$

We call $\boldsymbol{\Theta}$ a pre-dual system for $\boldsymbol{\Phi}$ if only the first property is satisfied. A pre-dual system can be turned into a dual system by scaling provided that one can show that

$$
\left(\theta_{P}, \phi_{P}\right) \neq 0 \quad \forall P .
$$

We make these distinctions only because we want to avoid the appearance of weird scaling factors, and prefer to work with $\theta_{P}$ that are normalized by

$$
\theta_{P}(P)=1 \quad \forall P .
$$

Following $[15,4]$, we restrict our attention to finding a dual system of the form

$$
\theta_{P}=\tilde{\phi}_{P}-\sum_{Q \in \tilde{\mathcal{V}}_{P}^{*}} \alpha_{P Q} \tilde{\phi}_{Q} \quad \forall P,
$$

where $\alpha_{P}=\left\{\alpha_{P Q}: Q \in \tilde{\mathcal{V}}_{P}^{*}\right\}$ needs to be determined. Obviously, these $\theta_{P}$ automatically satisfy (5).

The following proposition is known for semiregular dyadic refinement $[15](d \leq 3)$, and we claim that it also holds for the irregular case and $d=1,2$. This claim will be fully established for $d=1$ while for $d=2$ the proof is incomplete.

Proposition 1. There is a unique $\alpha_{P}$ such that the system $\Theta$ of the form (6) is pre-dual. Moreover, we have

$$
\left(\theta_{P}, \phi_{P}\right)=\left(\theta_{P}, 1\right) \asymp\left\|\theta_{P}\right\|_{p}^{p} \asymp \tilde{\Delta}_{P},
$$

i.e., $\boldsymbol{\Theta}$ is dual to $\boldsymbol{\Phi}$.

Proof. For $d=1$ we have $k=k_{P}=2$ for all $P$ in $\mathcal{V}$, and a simple calculation gives

$$
\begin{aligned}
\theta_{P} & =\phi_{P}-\frac{\left(\phi_{P}, \phi_{P^{-}}\right)}{\left(\tilde{\phi}_{Q^{-}}, \phi_{P^{-}}\right)} \tilde{\phi}_{Q^{-}}-\frac{\left(\phi_{P}, \phi_{P^{+}}\right)}{\left(\tilde{\phi}_{Q^{+}}, \phi_{P^{+}}\right)} \tilde{\phi}_{Q^{+}} \\
& =\tilde{\phi}_{P}-\alpha\left(t^{-}\right) \tilde{\phi}_{Q^{-}}-\alpha\left(t^{+}\right) \tilde{\phi}_{Q^{+}},
\end{aligned}
$$

where the auxiliary function $\alpha(t)$ is given by

$$
0 \leq \alpha(t):=\frac{t^{2}}{(1+t)} \leq \frac{1}{2}, \quad 0 \leq t \leq 1 .
$$

Here, $Q^{ \pm}$denote the two new vertices inserted into the intervals to the left $(-)$and right $(+)$ of $P$ at distance $t^{ \pm} d^{ \pm}$, where $d^{ \pm}=\tilde{\Delta}_{Q^{ \pm}}$is the length of the corresponding intervals in $\mathcal{T}$. Similarly, $P^{ \pm}$denote the old vertices immediately to the left resp. right of $P$. Note that $t^{ \pm}=1 / 2$ and thus $\theta_{P}\left(Q^{ \pm}\right)=-1 / 6$ for semiregular refinement. 
With this, (7) is immediate. Indeed,

$$
\begin{aligned}
\left(\theta_{P}, 1\right) & =\frac{1}{2}\left(\tilde{\Delta}_{P}-\alpha\left(t^{-}\right) \tilde{\Delta}_{Q^{-}}-\alpha\left(t^{+}\right) \tilde{\Delta}_{Q^{+}}\right) \\
& =\frac{1}{2}\left(\left(t^{-}-\alpha\left(t^{-}\right)\right) d^{-}+\left(t^{+}-\alpha\left(t^{+}\right)\right) d^{+}\right) \\
& =\frac{t^{-} d^{-}}{2\left(1+t^{-}\right)}+\frac{t^{+} d^{+}}{2\left(1+t^{+}\right)} \asymp\left(t^{-} d^{-}+t^{+} d^{+}\right)=\tilde{\Delta}_{P},
\end{aligned}
$$

with optimal constants $1 / 4$ and $1 / 2$ in the two-sided inequality. The $L_{p}$ norm of $\theta_{P}$ can be estimated in a similar fashion:

$$
\begin{aligned}
\left\|\theta_{P}\right\|_{p}^{p} & \asymp \tilde{\Delta}_{P}+\alpha\left(t^{-}\right)^{p} \tilde{\Delta}_{Q^{-}}+\alpha\left(t^{+}\right)^{p} \tilde{\Delta}_{Q^{+}} \\
& =\left(1+\frac{\left(t^{-}\right)^{2 p-1}}{\left(1+t^{-}\right)^{p}}\right) t^{-} d^{-}+\left(1+\frac{\left(t^{+}\right)^{2 p-1}}{\left(1+t^{+}\right)^{p}}\right) t^{+} d^{+} \asymp \tilde{\Delta}_{P} .
\end{aligned}
$$

Constants may depend on $p$, at most.

For $d=2$, uniqueness and pre-duality follow from the fact that the $k \times k$ matrix

$$
A=\left(\left(a_{l m}:=\left(\phi_{P_{l}}, \tilde{\phi}_{Q_{m}}\right), l, m=1, \ldots, k\right)\right)
$$

is non-singular (for the notation, see Fig. 1 a)). Indeed, we can then uniquely determine $\alpha_{P}$ by solving the linear system $A \alpha_{P}=b$, where $b$ is the column vector with the entries $b_{l}=\left(\phi_{P_{l}}, \tilde{\phi}_{P}\right), l=1, \ldots, k$. This system is equivalent to $\left(\theta_{P}, \phi_{P_{l}}\right)=0, l=1, \ldots, k_{P}$. For the remaining $P^{\prime} \neq P$ which are not among the immediate neighbors $P_{l}$ of $P$ we automatically have $\left(\theta_{P}, \phi_{P^{\prime}}\right)=0$ since $\theta_{P}(x) \phi_{P^{\prime}}(x) \equiv 0$ by the support properties. This implies that the system $\left\{\theta_{P}\right\}$ from (6) is pre-dual.

The non-singularity of $A$ follows from the fact that $A$ is columnwise diagonally dominant. To show this, observe that each column of $A$ contains exactly 3 positive non-zero elements. In the $m$-th column, these are $\left(\phi_{P_{l}}, \tilde{\phi}_{Q_{m}}\right)$ for $l=m-1, m, m+1$ (with obvious modifications if $m=1$ or $m=k$ ). Since

$$
\begin{aligned}
\delta_{m} & :=\left(\phi_{P_{m}}, \tilde{\phi}_{Q_{m}}\right)-\left(\phi_{P_{m-1}}, \tilde{\phi}_{Q_{m}}\right)-\left(\phi_{P_{m+1}}, \tilde{\phi}_{Q_{m}}\right) \\
& =\underbrace{\int_{\Delta P P_{m} P_{m+1}}\left(\phi_{P_{m}}-\phi_{P_{m+1}}\right) \tilde{\phi}_{Q_{m}} d x}_{=: \delta_{m}^{+}}+\underbrace{\int_{\Delta P P_{m} P_{m-1}}\left(\phi_{P_{m}}-\phi_{P_{m-1}}\right) \tilde{\phi}_{Q_{m}} d x}_{=: \delta_{m}^{-}},
\end{aligned}
$$

we establish diagonal dominance if we show that $\delta_{m}^{+}>0$ for an arbitrary $m$, independently of the geometry of the subdivided triangle $\Delta P P_{m} P_{m+1}$ (by symmetry arguments this implies that $\delta_{m}^{-}>0$ as well).

Without loss of generality, we will do this for $m=1$, and use the notation of Figure 2. Note that the parameters $x, y, z$ denote the relative distance (i.e., distance normalized by the length of the corresponding coarse edge) of $Q_{1}$ to $P$, of $Q_{2}$ to $P$, and of $Q_{1}^{\prime}$ to $P_{1}$, respectively. Relative distances of a $Q$ to its two related coarse grid vertices always sum up to 1 . What we are able to show is the following lower bound

$$
\delta_{1}^{+}>\frac{\Delta_{1}}{12}
$$

where the numbers $\Delta_{i}, i=1, \ldots, 4$, stand for the areas of the 4 subtriangles, compare 


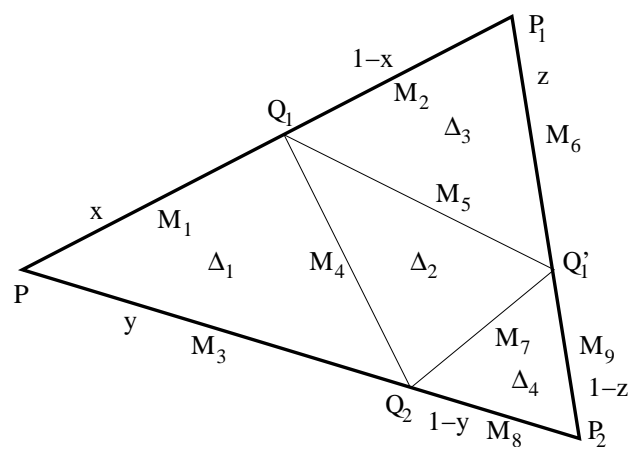

Fig. 2. Notation for the proof of (9)

Figure 2. Note that $\Delta_{1}=x y \Delta, \Delta_{3}=(1-x) z \Delta, \Delta_{4}=(1-y)(1-z) \Delta$, and $\Delta_{2}=$ $\Delta-\Delta_{1}-\Delta_{3}-\Delta_{4}$.

Since the integrand of the integral representing $\delta_{1}^{+}$is a quadratic polynomial on each of the subtriangles, we can apply the midpoint rule on each of them. It is easy to see that the nodal basis function $\tilde{\phi}_{Q_{1}}$ vanishes only at 4 of those midpoints (namely, $M_{1}, M_{2}, M_{4}$, and $M_{5}$, where it takes the value $1 / 2$ ). A straightforward calculation gives the following values of the linear function $g:=\phi_{P_{1}}-\phi_{P_{2}}$ at these points:

$$
g\left(M_{1}\right)=\frac{x}{2}, \quad g\left(M_{2}\right)=\frac{x+1}{2}, \quad g\left(M_{4}\right)=\frac{x-y}{2}, \quad g\left(M_{5}\right)=\frac{x+1-2 z}{2} .
$$

This leads to (we drop the trivial steps of calculating and simplifying the expressions)

$$
\begin{aligned}
\delta_{1}^{+} & =\frac{\Delta_{1}}{12}(2 x-y)+\frac{\Delta_{2}}{12}(2 x-y+1-2 z)+\frac{\Delta_{3}}{12}(2 x+2-2 z) \\
& =\frac{\Delta}{12}((2 x-y) x y+(2 x-y+1-2 z)(y+x z-x y-z y)+(2 x+2-2 z)(1-x) z) \\
& =\frac{\Delta}{12}\left(y+2 z-y^{2}-2 z^{2}+x y+x z-x y z+z y^{2}+2 z^{2} y-3 z y\right) \\
& =\frac{\Delta}{12}(x y+(1-y)(y(1-z)+2 z(1-z)+x z)) \\
& >\frac{x y \Delta}{12}=\frac{\Delta_{1}}{12}>0, \quad 0<x, y, z<1 .
\end{aligned}
$$

Thus, the strict columnwise diagonal dominance of $A$ has been established.

The open question for $d=2$ is whether the pre-dual system found can be turned into a dual system resp. whether the stronger statement (7) holds. Numerical evidence indicates that this statement holds with moderate constants, probably in the form

$$
\frac{\tilde{\Delta}_{P}}{9} \leq\left(\theta_{P}, \phi_{P}\right)=\left(\theta_{P}, 1\right) \leq \frac{\tilde{\Delta}_{P}}{3} .
$$

Similar bounds seem to hold for the $L_{p}$-norms of the $\theta_{P}$ (we have only collected numerical data for $\left.\left\|\theta_{P}\right\|_{2}^{2}\right)$. We also found numerically that $\alpha_{P Q_{m}}$ can become negative but that $\left|\alpha_{P Q_{m}}\right|$ always is bounded well below 1 (the numerical experiments consisted in generating thousands of randomly shaped and randomly subdivided 1-ring neighborhoods of the origin). 

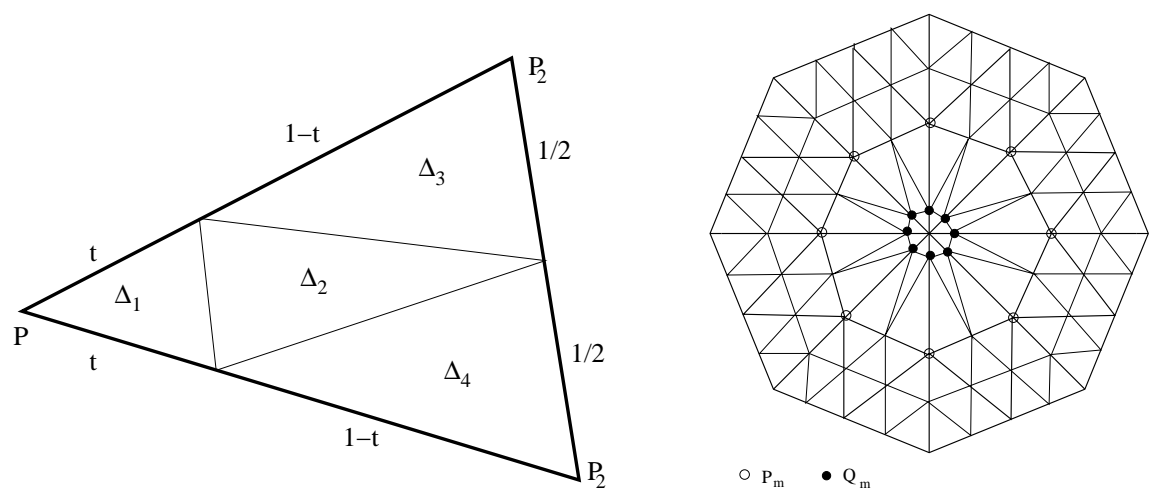

Fig. 3. Triangle of special 1-ring refinement (left), and triangulation for counterexamples $(k=8$, right)

In partial cases, e.g., for semiregular dyadic refinement, $\alpha_{P}$ can be found explicitly, which allows to verify (7) directly. As an example (which will be used to construct counterexamples later), we consider a special 1-ring where all new vertices $Q_{m}$ are inserted at the same relative distance $0<t<1$ from $P$, while the new vertices $Q_{m}^{\prime}$ coincide with the midpoints of the respective edges. This corresponds to the parameter choices $x=y=t$ and $z=1 / 2$ in our above calculations. The case $t=1 / 2$ corresponds to semiregular refinement. See Figure 3 (left) for a graphical depiction of the situation (only the triangle $\triangle P P_{1} P_{2}$ is shown). Under these assumptions, we find that $\alpha_{P}$ coincides with a constant vector, more precisely,

$$
\alpha_{P Q_{m}}=\tilde{\alpha}(t):=\frac{t^{3}}{1+t+t^{2}}, \quad m=1, \ldots, k_{P} .
$$

Note that for $t=1 / 2$ we get the known value of $\alpha_{P Q_{m}}=1 / 14$. By symmetry and rotational invariance, the above claim reduces to verifying that we have

$$
I:=\int_{\triangle P P_{1} P_{2}} \theta_{P} \phi_{1} d x=0
$$

for the above constant vector $\alpha_{P}$. This can be done by computing the values of $\theta_{P}$ and of $\phi_{1}$ at the edge midpoints for all 4 subtriangles, and applying the midpoint rule. The areas of the subtriangles are $\Delta_{1}=t^{2} \Delta, \Delta_{3}=\Delta_{4}=(1-t) \Delta / 2$, and $\Delta_{2}=t(1-t) \Delta$. We spare the reader these trivialities.

For short, set $\alpha:=\tilde{\alpha}(t)$. Using the same midpoint rule, we compute

$$
\begin{aligned}
\int_{\Delta P P_{1} P_{2}} \theta_{P} d x & =\frac{\Delta}{3}\left((1-2 \alpha) t^{2}-2 \alpha \frac{1-t}{2}-2 \alpha t(1-t)\right) \\
& =\frac{\Delta}{3}\left(t^{2}-\alpha(1+t)\right)=\frac{t^{2} \Delta}{3\left(1+t+t^{2}\right)} \asymp \Delta_{1},
\end{aligned}
$$

where the final two-sided inequality holds with constants $1 / 9$ and $1 / 3$, resp., for arbitrary $0<t<1$. Summing the similar expressions for all triangles $\Delta P P_{m} P_{m+1}$, we clearly get

$$
\frac{\tilde{\Delta}_{P}}{9} \leq\left(\theta_{P}, \phi_{P}\right)=\left(\theta_{P}, 1\right) \leq \frac{\tilde{\Delta}_{P}}{3} .
$$

Estimates for the $L_{p}$ norms of $\theta_{P}$ can be established analogously. 
4. Stability estimates. Given $\boldsymbol{\Phi}$ and its dual system $\boldsymbol{\Theta}$, a straightforward definition of a semiorthogonal prewavelet system $\boldsymbol{\Psi}=\left\{\psi_{Q}\right\}$ in $\tilde{V}$ is to set

$$
\psi_{Q}:=\tilde{\phi}_{Q}-\sum_{P \in \mathcal{V}} \beta_{Q P} \theta_{P}, \quad \beta_{Q P}:=\frac{\left(\tilde{\phi}_{Q}, \phi_{P}\right)}{\left(\theta_{P}, \phi_{P}\right)}=-\psi_{Q}(P), P \in \mathcal{V},
$$

where $Q \in \tilde{\mathcal{V}} \backslash \mathcal{V}$, see [15].

From now on, we restrict our attention to the special $\Theta$ given by (6), and considered in the previous section. In this case, for each $Q$ only finitely many $\beta_{Q P}$ are non-zero, which implies the desired local support properties of the $\psi_{Q}$. For $d=1$, supp $\psi_{Q}$ consists of 3 intervals from $\mathcal{T}$ (the one containing $Q$ and its two neighbors $Q^{ \pm}$), which is obviously minimal. Similarly, for $d=2$ we have

$$
\operatorname{supp} \psi_{Q} \subset \bigcup_{P \in \mathcal{V}_{Q}} \operatorname{supp} \theta_{P}
$$

(this construction does not lead to the smallest possible support, compare $[9,5]$ ).

The results reported in this section are conditional for $d=2$, since we will heavily rely on the existence and properties of $\boldsymbol{\Theta}$ stated in Proposition 1 which have been fully established only for $d=1$. We start with the upper stability estimate.

Proposition 2. Let $d=1,2$. Assume that the pre-dual system $\boldsymbol{\Theta}$ satisfies (6) and (4) (the latter property is not yet established if $d=2$ ), and that $\boldsymbol{\Psi}$ is defined as in (10). Then for $1 \leq p<\infty$ and arbitrary sequences $\left\{x_{Q}: Q \in \tilde{\mathcal{V}} \backslash \mathcal{V}\right\}$, we have

$$
\left\|\sum_{Q} x_{Q} \psi_{Q}\right\|_{p}^{p} \leq C_{p}\left(\sup _{P} k_{P}\right)^{p-1} \sum_{Q}\left|x_{Q}\right|^{p}\left\|\psi_{Q}\right\|_{p}^{p} .
$$

The dependence of the bound on the values of $k_{P}^{p-1}$ is essential for $d=2$, for $d=1$ it can be dropped since $k_{P}=2$ for all $P$.

Proof. Let $d=2(d=1$ is similar, see also below). By construction, on any triangle from $\mathcal{T}$ with vertices $P_{1}, P_{2}, P_{3}$ less than $2\left(k_{P_{1}}+k_{P_{2}}+k_{P_{3}}\right) \leq 6 \sup _{P} k_{P}$ prewavelets $\psi_{Q}$ do not vanish (more precisely, these are the functions $\psi_{Q}$ with $Q \in \tilde{\mathcal{V}}_{P_{1}} \cup \tilde{\mathcal{V}}_{P_{2}} \cup \tilde{\mathcal{V}}_{P_{3}}$ ). Thus, we have a pointwise estimate

$$
\left|\sum_{Q} x_{Q} \psi_{Q}\right|^{p} \leq\left(6 \sup _{P} k_{P}\right)^{p-1} \sum_{Q}\left|x_{Q}\right|^{p}\left|\psi_{Q}\right|^{p}
$$

from which (11) follows by integration. The counterexample will be given at the end of this section.

The proof of a lower stability estimate is a bit more involved, and requires the property (7) of $\boldsymbol{\Theta}$. We need the following lemma.

Lemma 3. Suppose $\boldsymbol{\Theta}$ satisfies the properties formulated in Proposition 1.

a) The coefficients $\beta_{P Q}$ defined in (10) satisfy

$$
\beta_{Q P} \asymp \frac{\left(\tilde{\phi}_{Q}, \phi_{P}\right)}{\tilde{\Delta}_{P}} \leq \tilde{\Delta}_{Q} / \tilde{\Delta}_{P}, \quad P \in \mathcal{V}_{Q}
$$


b) The prewavelets $\psi_{Q}$ from (10) satisfy

$$
\left\|\psi_{Q}\right\|_{p}^{p} \asymp \tilde{\Delta}_{Q}+\sum_{P \in \mathcal{V}_{Q}} \beta_{Q P}^{p} \tilde{\Delta}_{P}
$$

Proof. a) is an obvious consequence of the definitions (6), (10), and the property (7) of the dual functions. Concerning b), observe that $\psi_{Q}$ is the sum of $\leq 5$ terms. This, together with (7), gives the upper bound

$$
\left\|\psi_{Q}\right\|_{p}^{p} \leq 5^{p-1}\left(\left\|\tilde{\phi}_{Q}\right\|_{p}^{p}+\sum_{P \in \mathcal{V}_{Q}} \beta_{Q P}^{p}\left\|\theta_{P}\right\|_{p}^{p}\right) \leq C\left(\tilde{\Delta}_{Q}+\sum_{P \in \mathcal{V}_{Q}} \beta_{Q P}^{p} \tilde{\Delta}_{P}\right) .
$$

For the lower bound, note that by construction $\psi_{Q}(P)=-\beta_{Q P}$ for $P \in \mathcal{V}_{Q}$. Using the $L_{p}$-stability (2) of $\tilde{\boldsymbol{\Phi}}$ for $\psi_{Q}=\sum_{R} \psi_{Q}(R) \tilde{\phi}_{R}$, this gives

$$
\sum_{P \in \mathcal{V}_{Q}} \beta_{Q P}^{p} \tilde{\Delta}_{P} \leq C\left\|\psi_{Q}\right\|_{p}^{p}
$$

For the remaining term $\tilde{\Delta}_{Q}$, we proceed as follows. Again using (2) and then (10), we have

$$
\tilde{\Delta}_{Q} \leq C\left\|\tilde{\phi}_{Q}\right\|_{p}^{p} \leq C 5^{p-1}\left(\left\|\psi_{Q}\right\|_{p}^{p}+\sum_{P \in \mathcal{V}_{Q}} \beta_{Q P}^{p}\left\|\theta_{P}\right\|_{p}^{p}\right) .
$$

According to $(7)$, we can replace $\left\|\theta_{P}\right\|_{p}^{p}$ by its upper bound $C \tilde{\Delta}_{P}$. Together with the previous estimate, this implies the lower bound in b).

Proposition 4. Suppose $\boldsymbol{\Theta}$ satisfies the properties formulated in Proposition 1, and that $\boldsymbol{\Psi}$ is defined as in (10). Then for $1 \leq p<\infty$ and arbitrary sequences $\left\{x_{Q}: Q \in \tilde{\mathcal{V}} \backslash \mathcal{V}\right\}$, we have

$$
\sum_{Q}\left|x_{Q}\right|^{p}\left\|\psi_{Q}\right\|_{p}^{p} \leq C\left(1+\sup _{Q}\left(\max _{P \in \mathcal{V}_{Q}} \beta_{Q P}^{p-1}\right)\right)\left\|\sum_{Q} x_{Q} \psi_{Q}\right\|_{p}^{p} .
$$

The dependence of the constant on the values $\beta_{Q P}^{p-1}$ cannot be neglected.

Proof. We concentrate on $d=2$ (a stronger result for $d=1$ is given below). By Lemma 3 we have

$$
\begin{aligned}
\sum_{Q}\left|x_{Q}\right|^{p}\left\|\psi_{Q}\right\|_{p}^{p} & \leq C\left(\sum_{Q}\left|x_{Q}\right|^{p} \tilde{\Delta}_{Q}+\sum_{Q}\left|x_{Q}\right|^{p} \sum_{P \in \mathcal{V}_{Q}} \beta_{Q P}^{p} \tilde{\Delta}_{P}\right) \\
& \leq C \sum_{Q}\left|x_{Q}\right|^{p} \tilde{\Delta}_{Q}\left(1+\sum_{P \in \mathcal{V}_{Q}} \beta_{Q P}^{p-1}\right) \leq C\left(1+\sup _{Q} \max _{P} \beta_{Q P}^{p-1}\right)\|\tilde{g}\|_{p}^{p},
\end{aligned}
$$

where in the last step (2) has been applied to $\tilde{g}:=\sum_{Q} x_{Q} \tilde{\phi}_{Q} \in \tilde{V}$. The estimation of $\|\tilde{g}\|_{p}^{p}$ is similar to what we did for the lower bound in Lemma $\left.3 \mathrm{~b}\right)$ : Since $\tilde{g}(P)=0$, we can write

$$
\tilde{g}=g+\sum_{P}\left(\sum_{Q \in \tilde{\mathcal{V}}_{P}} x_{Q} \beta_{Q P}\right) \theta_{P}=g-\sum_{P} g(P) \theta_{P},
$$

where $g=\sum_{Q} x_{Q} \psi_{Q}$. In the expression on the right-hand side of this equality at most 4 terms are non-zero on any coarse triangle from $\mathcal{T}$. Therefore, we obtain

$$
\|\tilde{g}\|_{p}^{p} \leq 4^{p-1}\left(\|g\|_{p}^{p}+\sum_{P}|g(P)|^{p}\left\|\theta_{P}\right\|_{p}^{p}\right) \leq C\left(\|g\|_{p}^{p}+\sum_{P}|g(P)|^{p} \tilde{\Delta}_{P}\right) \leq C\|g\|_{p}^{p}
$$

where in the last two steps (7) and (2) were used. This shows (12). 
For $d=2$, the counterexamples for Proposition 2 and 4 are based on the special, parameter-dependent refinement of a 1-ring around a vertex $P$ discussed in Section 3 . Figure 3 (right) shows the essential portion of a special triangulation $\tilde{\mathcal{T}}$, with a vertex $P$ of valence $k$ in the center. The valences of the vertices $P_{m}$ in the 1-ring around $P$ are 6 . How $\tilde{\mathcal{T}}$ looks outside the shown portion is not essential for the result. For simplicity, we assume $k$-fold rotational symmetry w.r.t. the center $P$. The new vertices are inserted at the edge midpoints, only the points $Q_{m}$ on the edges emanating from $P$ are inserted at relative distance $0<t<1$ from $P$. All shown coarse triangles from $\mathcal{T}$ are similar, with two sides of length $k^{1 / 2}$ and the short side of length $\asymp k^{-1 / 2}$. Consider the prewavelets $\psi_{Q_{m}}$. These functions are "rotational" copies of $\psi_{Q_{1}}$. The existence of $\psi_{Q_{1}}$ can be shown by direct examination. To this end, we have to verify (7) at $P$ (which was already done in Section 3), and at $P_{m}$ which we leave to the reader.

Note that by construction of the triangulation, all coarse triangles have area $\asymp 1$. Thus, given the described placement of the $Q$ points, we have

$$
\tilde{\Delta}_{P} \asymp k t^{2}, \quad \tilde{\Delta}_{R} \asymp 1 \quad(R \neq P) .
$$

From Lemma 3 we therefore obtain $\beta_{Q P} \leq C k^{-1} t^{-2}$, and, thus,

$$
\left\|\psi_{Q_{m}}\right\|_{p}^{p} \asymp\left(1+\left(k t^{2}\right)^{-(p-1)}\right) .
$$

The lower bound requires that we in addition verify $\beta_{Q_{m} P} \asymp k^{-1} t^{-2}$ (start from Lemma $3 a)$ ).

Thus, if we choose $t=k^{-1 / 2}$, and define the (finitely supported) sequence $\left\{x_{Q}\right\}$ by setting $x_{Q}=1$ if $Q=Q_{m}, m=1, \ldots, k$, and $x_{Q}=0$ otherwise, we have

$$
\sum_{Q}\left|x_{Q}\right|^{p}\left\|\psi_{Q}\right\|_{p}^{p} \asymp k \text {. }
$$

On the other hand, because of rotational symmetry $g=\sum_{Q} x_{Q} \psi_{Q}$ has value

$$
|g(P)|=k\left|\psi_{Q_{1}}(P)\right|=k \beta_{Q_{1} P} \asymp k\left(\tilde{\phi}_{Q_{1}}, \phi_{P}\right) \asymp k .
$$

Using (2), this yields

$$
\|g\|_{p}^{p} \geq c|g(P)|^{p} \tilde{\Delta}_{P} \geq c k^{p} .
$$

Thus, comparing this lower bound with (14), we see that the constant in the upper stability estimate (11) stated in Proposition 2 cannot be smaller in order than $k^{p-1}$. This shows that the valence-dependence of the constant in (11) is essential.

To prove a similar result for the lower bound (12) of Proposition 4, we fix in the above construction $k$, and consider $g:=\psi_{Q_{1}}-\psi_{Q_{2}}$ for $t \rightarrow 0$. From (13) we have for $0<t<k^{-1 / 2}$ that $\beta_{Q_{m} P} \asymp k^{-1} t^{-2}$. Thus, the left-hand side in (12) is of the order $\beta_{Q_{1} P}^{p-1} \asymp\left(k t^{2}\right)^{-(p-1)}$. On the other hand, since the term involving $\theta_{P}$ cancels by definition of $g$, we can represent $g$ as

$$
\begin{aligned}
g= & \tilde{\phi}_{Q_{1}}-\tilde{\phi}_{Q_{2}}-\beta_{Q_{1} P_{k}} \theta_{P_{k}}+\beta_{Q_{2} P_{3}} \theta_{P_{3}} \\
& +\left(\beta_{Q_{2} P_{1}}-\beta_{Q_{1} P_{1}}\right) \theta_{P_{1}}+\left(\beta_{Q_{2} P_{2}}-\beta_{Q_{1} P_{2}}\right) \theta_{P_{2}} .
\end{aligned}
$$

All coefficients in this representation (and the $L_{p}$-norms of the involved functions $\tilde{\phi}_{Q}$ and $\left.\theta_{P^{\prime}}\right)$ are bounded by some constant $C$ which gives $\|g\|_{p}^{p} \leq C$, independently of $t$. 
Comparing these findings with (12), we see that in our particular case the constant there is bounded from below by $c \beta_{Q_{m} P}^{p-1}$. Thus, the appearance of such terms in the constant for the lower stability estimate (12) is essential.

For $d=1$, we have stronger results. Obviously, in Lemma 3 we can now establish

$$
\beta_{Q P} \asymp \frac{\tilde{\Delta}_{Q}}{\tilde{\Delta}_{P}}, \quad P \in \mathcal{V}_{Q}, \quad\left\|\psi_{Q}\right\|_{p}^{p} \asymp \tilde{\Delta}_{Q}\left(1+\sum_{P \in \mathcal{V}_{Q}} \beta_{Q P}^{p-1}\right) .
$$

Define the quantity

$$
\tau:=\sup _{P} \min _{Q \in \tilde{\mathcal{V}}_{P}} \beta_{Q P} \asymp \tau^{\prime}:=\sup _{P \in \mathcal{V}} \frac{\min \left(\tilde{\Delta}_{Q^{-}}, \tilde{\Delta}_{Q^{+}}\right)}{\tilde{\Delta}_{P}}
$$

(as before, $Q^{ \pm}$are the new vertices left and right from $P$ ). Note that $\tau^{\prime}$ is a somewhat nonstandard quantitative measure for the irregularity of the refinement from $\mathcal{T}$ to $\tilde{\mathcal{T}}$. It is easy to construct examples of partitions for which $\tau^{\prime} \asymp \tau$ is significantly smaller than the constant

$$
\bar{\tau}:=\sup _{P} \max _{Q \in \tilde{\mathcal{V}}_{P}} \beta_{Q P},
$$

that entered the estimate given in Proposition 4. Note that $\tau, \tau^{\prime} \geq c>0$ for some absolute constant $c$.

Proposition 5. Let $d=1$. Suppose that $\Theta$ and $\boldsymbol{\Psi}$ are defined by (6) resp. (10). Then the $L_{p}$-condition of $\boldsymbol{\Psi}$ satisfies

$$
\kappa_{p}(\boldsymbol{\Psi}) \asymp \tau^{p-1}, \quad 1 \leq p<\infty,
$$

with constants independent of $\mathcal{T}, \tilde{\mathcal{T}}$.

Proof. For the upper estimate of $\kappa_{p}(\boldsymbol{\Psi})$ we need the following improvement of Proposition 4: For $g \in \tilde{V}$ of the form $g=\sum_{Q} x_{Q} \psi_{Q}$, we have

$$
\sum_{Q}\left|x_{Q}\right|^{p}\left\|\psi_{Q}\right\|_{p}^{p} \leq C \tau^{p-1}\|g\|_{p}^{p} .
$$

The counterpart of Proposition 2 for $d=1$ is

$$
\|g\|_{p}^{p} \leq 3^{p-1} \sum_{Q}\left|x_{Q}\right|^{p}\left\|\psi_{Q}\right\|_{p}^{p}
$$

(because in the sum $\sum_{Q} x_{Q} \psi_{Q}$ at most 3 terms are non-zero for any argument). These two inequalities give the upper bound in (17).

The proof of (18) heavily relies on the following recovery formula for the coefficients $x_{Q}$ of $g=\sum_{Q} x_{Q} \psi_{Q}$ :

$$
x_{Q}=\lambda_{Q}(g):=g(Q)+\alpha_{P^{-} Q} g\left(P^{-}\right)+\alpha_{P^{+} Q} g\left(P^{+}\right),
$$

where $\ldots, Q^{-}, P^{-}, Q, P^{+}, Q^{+}, \ldots$ denote the vertices immediately to the left and right from $Q$. The values $\alpha_{P^{ \pm} Q}$ are defined by (6) and (8), and belong to [0,1/2]. The reader can easily verify this expression for $x_{Q}$ by using the explicit formulas for the nodal values of the prewavelets which follow from the definitions (10), (6), and (8):

$$
\psi_{Q}\left(P^{ \pm}\right)=-\beta_{Q P^{ \pm}}, \quad \psi_{Q}\left(Q^{ \pm}\right)=\beta_{Q P^{ \pm}} \alpha_{P^{ \pm} Q^{ \pm}},
$$


and $\psi_{Q}(Q)=1+\beta_{Q P^{-}} \alpha_{P^{-} Q}+\beta_{Q P^{+}} \alpha_{P^{+} Q}$. Using (20), we obtain $\lambda_{Q}\left(\psi_{Q}\right)=1$ and $\lambda_{Q}\left(\psi_{Q^{ \pm}}\right)=0$ (on all other $\psi_{Q^{\prime}}$ the functional $\lambda_{Q}$ vanishes trivially due to the support properties of $\psi_{Q^{\prime}}$ ). This implies (19).

As a preparation, we will show that

$$
\|g\|_{p}^{p} \asymp \sum_{Q}\left|x_{Q}\right|^{p} \tilde{\Delta}_{Q}+\sum_{P}|g(P)|^{p} \tilde{\Delta}_{P} .
$$

Indeed, by definition of $\alpha_{P^{ \pm} Q}$ via (6) and (8) we have

$$
\alpha_{P^{ \pm} Q} \leq \min \left(\frac{1}{2},\left(\frac{\tilde{\Delta}_{P^{ \pm}}}{\tilde{\Delta}_{Q}}\right)^{2}\right)
$$

and in conjunction with (19)

$$
\begin{aligned}
\left|x_{Q}\right|^{p} \tilde{\Delta}_{Q} & \leq\left(1+\alpha_{P^{-}}^{1-\frac{1}{2 p}}+\alpha_{P+Q}^{1-\frac{1}{2 p}}\right)^{p-1} \tilde{\Delta}_{Q}\left(|g(Q)|^{p}+\alpha_{P^{-} Q}^{\frac{1}{2}}\left|g\left(P^{-}\right)\right|^{p}+\alpha_{P^{+} Q}^{\frac{1}{2}}\left|g\left(P^{+}\right)\right|^{p}\right) \\
& \leq 3^{p-1}\left(|g(Q)|^{p} \tilde{\Delta}_{Q}+\left|g\left(P^{-}\right)\right|^{p} \tilde{\Delta}_{P^{-}}+\left|g\left(P^{+}\right)\right|^{p} \tilde{\Delta}_{P^{+}}\right) .
\end{aligned}
$$

Summation with respect to $Q$ and use of the $L_{p}$-stability of $\tilde{\boldsymbol{\Phi}}$ gives one direction in (21). The opposite inequality follows in the same way if (19) is rewritten as

$$
g(Q)=x_{Q}-\alpha_{P^{-} Q} g\left(P^{-}\right)-\alpha_{P^{+} Q} g\left(P^{+}\right),
$$

and used to estimate the terms $|g(Q)|^{p} \tilde{\Delta}_{Q}$.

We are now in a position to attack (18). Since by (15)

$$
\sum_{Q}\left|x_{Q}\right|^{p}\left\|\psi_{Q}\right\|_{p}^{p} \leq C \sum_{Q}\left|x_{Q}\right|^{p} \tilde{\Delta}_{Q}\left(1+\beta_{Q P^{-}}^{p-1}+\beta_{Q P^{+}}^{p-1}\right),
$$

it is enough to concentrate on the critical terms with $\beta_{Q P^{+}}>\tau$ resp. $\beta_{Q P^{-}}>\tau$ (the other terms are bounded by $\left.\leq C \tau^{p-1}\left|x_{Q}\right|^{p} \tilde{\Delta}_{Q}\right)$. E.g., let $\beta_{Q P^{+}}>\tau$ for some $Q$. By definition of $\tau$ we have $\beta_{Q^{+} P^{+}} \leq \tau$, and we can use the identity $g\left(P^{+}\right)=-\left(\beta_{Q P^{+}} x_{Q}+\beta_{Q^{+} P^{+}} x_{Q^{+}}\right)$ together with (15) to estimate as follows:

$$
\begin{aligned}
\beta_{Q P^{+}}^{p-1}\left|x_{Q}\right|^{p} \tilde{\Delta}_{Q} & =\left|\beta_{Q P^{+}}^{-1 / p}\left(g\left(P^{+}\right)+\beta_{Q^{+} P^{+}} x_{Q^{+}}\right)\right|^{p} \tilde{\Delta}_{Q} \\
& \leq C \beta_{Q P^{+}}^{-1}\left(\left|g\left(P^{+}\right)\right|^{p}+\beta_{Q^{+} P^{+}}^{p}\left|x_{Q^{+}}\right|^{p}\right) \tilde{\Delta}_{Q} \\
& \leq C\left(|g(P)|^{p} \tilde{\Delta}_{P^{+}}+\tau^{p-1}\left|x_{Q^{+}}\right|^{p} \tilde{\Delta}_{Q^{+}}\right) .
\end{aligned}
$$

With this estimate for the critical terms at hand, the overall result is

$$
\sum_{Q}\left|x_{Q}\right|^{p}\left\|\psi_{Q}\right\|_{p}^{p} \leq C\left(\tau^{p-1} \sum_{Q}\left|x_{Q}\right|^{p} \tilde{\Delta}_{Q}+\sum_{P}|g(P)|^{p} \tilde{\Delta}_{P}\right) \leq C \tau^{p-1}\|g\|_{p}^{p},
$$

where (21) was used. This gives (18), and the upper estimate for $\kappa_{p}(\boldsymbol{\Psi})$ in (17).

For the lower estimate, note that the stability constants $C_{1}, C_{2}$ in (1) must always satisfy $C_{1} \leq 1 \leq C_{2}$ (just take $x_{1}=1$, and $x_{i}=0$ for all $i \neq 1$ ). Thus, the result follows if we establish $C_{1} \leq C \tau^{1-p}$, or, equivalently, we find $g=\sum_{Q} x_{Q} \psi_{Q}$ such that

$$
\sum_{Q}\left|x_{Q}\right|^{p}\left\|\psi_{Q}\right\|_{p}^{p} \geq c \tau^{p-1}\|g\|_{p}^{p} .
$$


Moreover, it is enough to consider $\tau_{0} \leq \tau$, where $\tau_{0}$ is fixed but sufficiently large. By definition of $\tau$, there exists $P \in \mathcal{V}$ such that $\beta_{Q^{+} P}, \beta_{Q^{-P}} \geq \tau / 2$, where this time

$$
\ldots, Q_{1}^{-}, P^{-}, Q^{-}, P, Q^{+}, P^{+}, Q_{1}^{+}, \ldots
$$

denote the vertices immediately to the left and right from $P$ (the case $\tau=\infty$ requires some obvious modifications). Set

$$
g=\beta_{Q^{+} P^{-1}}^{-1} \psi_{Q^{+}}-\beta_{Q^{-} P}^{-1} \psi_{Q^{-}} .
$$

The coefficients $x_{Q^{ \pm}}$have been chosen such that $g(P)=0$, moreover, by (20) we compute $g\left(P^{+}\right)=\beta_{Q^{+} P^{+}} / \beta_{Q^{+} P}$. To get an estimate for $\left|g\left(P^{+}\right)\right|^{p}$, we use that $\tilde{\Delta}_{Q^{+}} / \tilde{\Delta}_{P} \geq c_{0} \beta_{Q^{+} P}$ holds for some $c_{0}>0$ according to (15). Thus,

$$
\tilde{\Delta}_{P^{+}}+\tilde{\Delta}_{P}>\tilde{\Delta}_{Q^{+}} \geq c_{0} \beta_{Q^{+}} \tilde{\Delta}_{P} \geq \frac{c_{0} \tau}{2} \tilde{\Delta}_{P},
$$

which gives for $\tau \geq \tau_{0}:=4 / c_{0}$

$$
\frac{\beta_{Q^{+} P^{+}}}{\beta_{Q^{+} P}} \leq C \frac{\tilde{\Delta}_{P}}{\tilde{\Delta}_{P^{+}}} \leq \frac{C}{c_{0} \tau / 2-1} \leq C \tau^{-1} .
$$

We conclude that

$$
\left|g\left(P^{+}\right)\right|^{p}=\left|\frac{\beta_{Q^{+} P^{+}}}{\beta_{Q^{+} P}}\right|^{p} \leq C \tau^{1-p} \frac{\tilde{\Delta}_{P}}{\tilde{\Delta}_{P^{+}}},
$$

analogously for $\left|g\left(P^{-}\right)\right|^{p}$. Substituting into (21), we obtain

$$
\begin{aligned}
\|g\|_{p}^{p} & \leq C\left(\beta_{Q^{+} P^{-p}} \tilde{\Delta}_{Q^{+}}+\beta_{Q^{-} P}^{-p} \tilde{\Delta}_{Q^{-}}+\left|g\left(P^{+}\right)\right|^{p} \tilde{\Delta}_{P^{+}}+\left|g\left(P^{-}\right)\right|^{p} \tilde{\Delta}_{P^{-}}\right) \\
& \leq C \tau^{1-p} \tilde{\Delta}_{P}, \quad \tau \geq \tau_{0} .
\end{aligned}
$$

Since $\tau \asymp \tau^{\prime}$ are bounded away from zero, this estimate holds (with another constant $C$ ) for small $\tau$, too.

On the other hand, by (15) we get

$$
\left|x_{Q^{+}}\right|^{p}\left\|\psi_{Q^{+}}\right\|_{p}^{p} \geq c \beta_{Q^{+} P}^{-p} \tilde{\Delta}_{Q^{+}} \beta_{Q^{+P}}^{p-1} \geq c \tilde{\Delta}_{P} .
$$

This gives the desired lower bound (22), and completes the proof of Proposition 5 .

Proposition 6. Let $\left\{\mathcal{T}_{j}, j \geq 0\right\}$ be a sequence of partitions of $\mathbb{R}^{1}$ obtained recursively by irregular dyadic refinement from $\mathcal{T}_{0}$. Denote by $\tau_{j} \asymp \tau_{j}^{\prime}$ the quantities defined in (16) with $\mathcal{T}=\mathcal{T}_{j-1}, \tilde{\mathcal{T}}=\mathcal{T}_{j}$, and by $\boldsymbol{\Psi}_{j}$ the associated semiorthogonal prewavelet system, $j \geq 1$. Let $\boldsymbol{\Psi}_{0}:=\boldsymbol{\Phi}_{0}$ be the system of hat functions with respect to $\mathcal{T}_{0}$. Then the multilevel prewavelet system $\left\{\boldsymbol{\Psi}_{0}, \boldsymbol{\Psi}_{1}, \boldsymbol{\Psi}_{2}, \ldots\right\}$, after normalization with respect to the $L_{2}$ norm, forms a Riesz basis in $L_{2}(\mathbb{R})$ if and only if $\sup _{j \geq 1} \tau_{j}^{\prime}<\infty$.

This result is an obvious consequence of the $L_{2}$-stability result of Proposition 5 applied to each $\boldsymbol{\Psi}_{j}$, and the mutual orthogonality between $\boldsymbol{\Psi}_{j}$ with different level indices $j$. This Riesz basis criterion could even be useful in practical terms since it allows to effectively control the Riesz bounds of the resulting prewavelet system via the ratios $\tilde{\Delta}_{Q^{ \pm}} / \tilde{\Delta}_{P}$ during the refinement process, i.e., when inserting new points. Moreover, the result induces a uniform condition number estimate for stiffness matrices if subsystems of the above multilevel prewavelet system are used to numerically solve symmetric $L_{2}$-elliptic variational problems by the Galerkin method. 
We conjecture that the above condition also characterizes the $L_{p}$-unconditionality $(1<p<\infty)$ of this multilevel prewavelet system. This needs to be contrasted with the $L_{p}$-unconditionality results for Franklin systems [7] which do not require any conditions on the refinement process. We do not know whether allowing larger support sizes for the $\psi_{Q}$ in the semiorthogonal case (or replacing semiorthogonality by biorthogonality, see [16]) would lead to the construction of $L_{p}$-stable locally supported prewavelet systems with no constraints on the irregular dyadic refinement. However, the main open problem is to close the gaps in the $2 \mathrm{D}$ case, where a full proof of the claims in Proposition 1 is missing.

\section{References}

[1] Z. Ciesielski and A. Kamont, Projections onto piecewise linear functions, Functiones and Approximation, Comment. Math. 25 (1997), 129-143.

[2] C. K. Chui and J. Z. Wang, On compactly supported wavelets and a duality principle, Trans. Amer. Math. Soc. 330 (1992), 903-915.

[3] C. K. Chui, J. Stöckler and J. Ward, Compactly supported box spline wavelets, Approx. Theory \& Appl. 8 (1992), 77-100.

[4] W. Dahmen and R. Stevenson, Element-by-element construction of wavelets satisfying stability and moment conditions, SIAM J. Numer. Anal. 37 (1999), 319-352.

[5] M. S. Floater and E. G. Quak, Piecewise linear prewavelets on arbitrary triangulations, Numer. Math. 82 (1999), 221-252.

[6] G. Gevorkyan and A. Kamont, On general Franklin systems, Diss. Math. 174 (1998).

[7] G. Gevorkyan and A. Kamont, Unconditionality of general Franklin systems in $L_{p}[0,1]$, $1<p<\infty$, Studia Math. 164 (2004), 161-204.

[8] R.-Q. Jia and C. A. Miccelli, Using the refinement equation for the construction of prewavelets II: power of two, in: Curves and Surfaces, P. J. Laurent, A. Mehaute, L. L. Schumaker (eds.), Acad. Press, New York, 1991, 209-246.

[9] U. Kotyczka and P. Oswald, Piecewise linear prewavelets of small support, in: Approximation Theory VIII, vol. 2: Wavelets and Multilevel Approximation, C. K. Chui, L. L. Schumaker (eds.), World Scientific, Singapore, 1995, 235-242.

[10] T. Lyche, K. Morken and E. Quak, Theory and algorithms for nonuniform spline wavelets, in: Multivariate Approximation and Applications, A. Pinkus, D. Leviatan, N. Dyn, D. Levin (eds.), Cambridge Univ. Press, 2001, 152-187.

[11] J. A. Mikkelsen, P. Oja and E. Quak, Stability of piecewise linear B-wavelets, preprint, 2001 (submitted).

[12] P. Oja and E. Quak, An example concerning the $L_{p}$-stability of piecewise linear B-wavelets, in: Algorithms for Approximation IV, I. Anderson and J. Levesley (eds.), 2002.

[13] P. Petrushev, Anisotropic spaces and nonlinear n-term spline approximation, in: 11th Int. Conf. Approximation Theory, Gatlinburg, TN, 2004 (to appear).

[14] S. Riemenschneider and Z. Shen, Wavelets and prewavelets in low dimensions, J. Approx. Th. 71 (1992), 18-38.

[15] R. Stevenson, Piecewise linear (pre-)wavelets on non-uniform meshes, in: Multigrid Methods V, W. Hackbusch and G. Wittum (eds.), Springer, Heidelberg, 1996, 306-319.

[16] R. Stevenson, Locally supported piecewise polynomial biorthogonal wavelets on nonuniform meshes, Constr. Approx. 19 (2003), 477-508. 